\title{
Distributed RSS-Based Localization in Wireless Sensor Networks with Node Selection Mechanism
}

\author{
Slavisa Tomic ${ }^{4(\bowtie)}$, Marko Beko ${ }^{1,3}$, Rui Dinis ${ }^{2,5}$, \\ Goran Dimic ${ }^{6}$, and Milan Tuba ${ }^{7}$ \\ ${ }^{1}$ Universidade Lusófona de Humanidades e Tecnologias, Lisbon, Portugal \\ mbeko@uninova.pt \\ ${ }^{2}$ DEE/FCT/UNL, Caparica, Portugal \\ rdinis@fct.unl.pt \\ ${ }^{3}$ UNINOVA - Campus FCT/UNL, Caparica, Portugal \\ ${ }^{4}$ ISR/ IST, Lisbon, Portugal \\ s.tomic@campus.fct.unl.pt \\ ${ }^{5}$ Instituto de Telecomunicações, Lisbon, Portugal \\ ${ }^{6}$ Institute Mihailo Pupin, University of Belgrade, Belgrade, Serbia \\ goran.dimic@pupin.rs \\ ${ }^{7}$ Faculty of Computer Science, Megatrend University, Belgrade, Serbia \\ tubalieee.org
}

\begin{abstract}
In this work, we address the target localization problem in large-scale cooperative wireless sensor networks (WSNs). Using the noisy range measurements, extracted from the received signal strength (RSS) information, we formulate the localization problem based on the maximum likelihood (ML) criterion. ML-based solutions are particularly important due to their asymptotically optimal performance, but the localization problem is highly non-convex. To overcome this difficulty, we propose a convex relaxation leading to secondorder cone programming (SOCP), which can be efficiently solved by interiorpoint algorithms. Furthermore, we investigate the case where target nodes limit the number of cooperating nodes by selecting only those neighbors with the highest RSS measurements. This simple procedure may decrease the energy consumption of an algorithm in both communication and computation phase. Our simulation results show that the proposed approach outperforms the existing ones in terms of the estimation accuracy. Moreover, they show that the new approach does not suffer significant degradation in its performance when the number of cooperating nodes is reduced.
\end{abstract}

Keywords: Wireless localization ' Wireless sensor network (WSN) · Received signal strength (RSS) - Second-order cone programming problem $(\mathrm{SOCP}) \cdot$ Cooperative localization $\cdot$ Distributed localization

\section{Introduction}

Ad hoc wireless sensor networks (WSNs) composed of tiny and low-power sensor nodes scattered over a region of interest form a very useful tool for monitoring the environmental characteristics, such as temperature, sound levels, air pollution, etc. Low device costs and savings in the infrastructure permit deployment of tens, 
hundreds, or even thousands of sensor nodes [1]. Besides sensing, nodes have a limited capability of processing and communicating the acquired data.

In many practical applications, data gathered inside a WSN are irrelevant if the locations of the reference nodes are not known. Hence, nodes' position information is a key requirement for many applications. Installing a global positioning system (GPS) device in each sensor node would be very expensive, and it would also restrict the network applicability [2]. An alternative and more cost-efficient solution is to equip only a small fraction of nodes with GPS, called anchor (or reference) nodes, and to determine the position of the remaining nodes, called target nodes, by employing the (noisy) ranging information between target and anchor nodes.

Ranging (distance) measurements are obtained between two sensor nodes which are in the communication range of each other, and are typically extracted from timeof-arrival (TOA), time-difference-of-arrival (TDOA), round-trip time (RTT), angleof-arrival (AOA), received signal strength (RSS) information or a combination of them, depending on the available hardware [3]. Ranging based on RSS has the lowest implementation costs, since it does not require any special hardware [3], rendering it an attractive low-cost solution for the localization problem.

The required processing inherent to localization schemes can be executed in a centralized or a distributed fashion. The former approach assumes the existence of a fusion center which coordinates the network and performs all computational processing. Moreover, this approach is characterized by stability and fundamental optimality, but its computational complexity grows with the number of nodes in the network [2]. On the other hand, the main advantages of the latter approach are scalability and lowcomplexity. However, this approach is executed iteratively; thus, it is sensitive to error propagation and might require a long convergence time. Hence, the trade-off between the computational complexity and estimation accuracy is the main feature that determines which approach is more suitable to employ. In this work, we will focus exclusively on the distributed algorithms, since we deal with large-scale WSNs, and this approach is more likely to preserve energy.

Recently, RSS-based localization techniques have attracted much attention in the research society [4]-[11]. The approaches described in [4]-[8] consider centralized algorithms exclusively. In [9], a distributed algorithm based on an augmented Lagrangian approach using primal-dual decomposition was presented. However, the authors dealt only with a non-cooperative localization problem, where a single target node emits beacon frames to all anchor nodes in the network. A distributed cooperative algorithm characterized by a spatial constraint that limits the solution space to a region around the current position estimate was presented in [10]. Using discretization of the solution space, the authors in [10] found the position update of each node by minimizing a local objective function over the candidate set using direct substitution. Another distributed cooperative localization algorithm that dynamically estimates the path loss exponent (PLE) by using RSS measurements was introduced in [11]. This algorithm was based on gradient descent search which employs a circular GaussSeidel algorithm. Although the approaches in [10], [11] have excellent computational characteristics, their performance highly depends on good initialization, since the objective function is non-convex and the algorithm may get trapped into local minima or a saddle point, resulting in a large estimation error. 
In this paper, we consider a large-scale RSS-based cooperative localization problem, and we propose a solution which is based entirely on a distributed approach. We derive a novel second-order cone programming (SOCP) estimator, which, in huge contrast to the existing methods, does not depend on initialization and requires much less iterations to converge. We also investigate the case where the target nodes limit the number of cooperating nodes (neighbors), discarding potentially bad links and reducing energy consumption in the network.

The remainder of this paper is structured as follows. In Section 2, a relationship of the research topic to cloud-based solutions is defined. In Section 3 the RSS measurement model is introduced and target localization problem is formulated. Section 4 provides details about the development of the proposed SOCP estimator and the node selection mechanism for reducing the number of cooperating nodes. The analysis of the computational complexity is summarized in Section 5. In Section 6 we provide the simulation results in order to compare the performance of the new approach and the existing ones. The main conclusions are summarized in Section 7.

\section{Relationship to Cloud-Based Solutions}

Sensor nodes in WSNs are deployed over a region of interest in order to obtain the desired information (such as temperature, noise pollution, etc.). Due to the limited power and computational potential of sensor nodes, one may consider connecting them to the "cloud". This approach channels the effort towards providing connectivity and communication capabilities to each sensor node by connecting them to the "cloud", rather than trying to increase local computational resources at each sensor node, which might be technically difficult for some cases. As a result, not only flexibility and more robust security mechanisms may be acquired, but also access to larger pools of resources available on the Internet can be enabled, allowing the development of higher value-added services, and faster and better decisions to each individual device to accompany the changes in the environment.

\section{$3 \quad$ Measurement Model}

We consider a WSN with $|\mathcal{T}|+|\mathcal{A}|$ nodes, where $\mathcal{T}$ and $\mathcal{A}$ are the sets of all target and anchor nodes, respectively, and $|\cdot|$ represents the cardinality of a set $(|\mathcal{T}|=$ $T,|\mathcal{A}|=A)$. The locations of the nodes are denoted as $x_{1}, \ldots, x_{T}, x_{T+1}, \ldots, x_{T+A}$, where $\boldsymbol{x}_{i} \in \mathbb{R}^{q}(q \geq 2)$. The considered network can also be seen as a connected graph, $\mathcal{G}(\mathcal{V}, \mathcal{E})$, where $\mathcal{V}$ and $\mathcal{E}$ are the set of vertices and edges, respectively. In order to preserve the energy and prolong the lifetime of the network, each node has a limited communication range, $R$. Hence, two nodes, $i$ and $j$, can exchange information if and only if they are within the communication range of each other, i.e., $\mathcal{E}=$ $\left\{(i, j):\left\|\boldsymbol{x}_{i}-\boldsymbol{x}_{j}\right\| \leq R, i \neq j\right\}$. Target node $i$ considers any neighboring node $j$ (target or anchor) as an anchor node in the localization process. Set of neighbors of a target node $i$ is defined as $\Omega_{i}=\{j:(i, j) \in \mathcal{E}\}$. 
For ease of expression, we define $\boldsymbol{X}=\left[\boldsymbol{x}_{1}, \boldsymbol{x}_{2}, \ldots, \boldsymbol{x}_{T}\right]$ as the $q \times T$ matrix of all target positions in the WSN. We assume that the anchor positions are known a priori, while each target node $i$ is given an initial estimation of its position, $\widehat{\boldsymbol{x}}_{i}^{(0)}, i=1, \ldots, T$; hence, $\widehat{\boldsymbol{X}}^{(0)}$ contains all initial estimations of the target positions.

From the relationship $L_{i j}=10 \log _{10} \frac{P_{T}}{P_{i j}}$, where $P_{T}$ is the transmit power of a node, and $P_{i j}$ is the received power at the $i$-th target node from the $j$-th neighboring node, the RSS localization problem can be formulated according to the path loss model (in dB) instead of RSS [12],[13]

$$
L_{i j}=L_{0}+10 \gamma \log _{10} \frac{\left\|\boldsymbol{x}_{i}-\boldsymbol{x}_{j}\right\|}{d_{0}}+n_{i j}, \forall(i, j) \in \mathcal{E},
$$

where $L_{0}$ is the path loss value at a short reference distance $d_{0}\left(\left\|x_{i}-x_{j}\right\| \leq d_{0}\right), \gamma$ is the path loss exponent, and $n_{i j}$ is the log-normal shadowing term between nodes $i$ and $j$, modeled as a zero-mean Gaussian random variable with variance $\sigma_{i j}^{2}$, i.e., $n_{i j} \sim \mathcal{N}\left(0, \sigma_{i j}^{2}\right)$. We assume that all path loss measurements are symmetric, i.e., $L_{i j}=$ $L_{j i}$, for $i \neq j$.

Based on the measurements from (1), we derive the maximum likelihood (ML) estimator as

$$
\widehat{\boldsymbol{X}}=\operatorname{argmin}_{\boldsymbol{X}} \sum_{(i, j):(i, j) \in \mathcal{E}} \frac{1}{\sigma_{i j}^{2}}\left[L_{i j}-L_{0}-10 \gamma \log _{10} \frac{\left\|\boldsymbol{x}_{i}-\boldsymbol{x}_{j}\right\|}{d_{0}}\right]^{2} .
$$

The least squares (LS) problem defined in (2) is non-linear and non-convex; hence, finding its globally optimal solution is difficult, since there may exist multiple local optima. In the following text we will show that the ML estimator in (2) can be approximated into a convex estimator, using SOCP relaxation, which can be solved efficiently by interior-point algorithms [14].

For the sake of simplicity (and without loss of generality), in the remainder of this work we assume that $\sigma_{i j}^{2}=\sigma^{2}, \forall(i, j) \in \mathcal{E}$. Moreover, we assume that $P_{T}$ of all nodes are identical, i.e., $L_{0}$ and $R$ are equivalent for all nodes.

\section{Distributed Algorithm}

Observe that the objective function in (2) depends only on the positions and pairwise measurements between the incident nodes. Given that the initial guess of the targets' positions and the true anchors' positions are known, problem in (2) can be solved independently by each target node, using only local information from its neighbors. Breaking down the localization problem in (2) into smaller sub-problems is particularly suitable for large-scale and highly-dense networks, because this approach can significantly reduce the computational complexity of an algorithm.

Solving the localization problem in a distributed fashion implies using an iterative scheme, consisting of two phases:

1) Communication phase - each target node in the network transmits its position estimate to its neighbors. 
2) Computation phase - each target node updates its position estimate. To update its position, target node $i$ solves the following optimization problem:

$$
\widehat{\boldsymbol{x}}_{i}^{(k+1)}=\underset{\boldsymbol{x}_{\mathrm{i}}}{\operatorname{argmin}} \sum_{(i, j) \in \mathcal{E}} \frac{1}{\sigma^{2}}\left[L_{i j}-L_{0}-10 \gamma \log _{10} \frac{\left\|\boldsymbol{x}_{i}-\widehat{\boldsymbol{x}}_{j}\right\|}{d_{0}}\right]^{2},
$$

Where $\widehat{\boldsymbol{x}}_{j}$ denotes the last position estimate of the $j$-th neighboring target node (or the true node position, if $j$-th node is an anchor) received by $i$-th target node. In the following text, we will show that (3) can be approximated into a convex problem.

\subsection{The Proposed SOCP Estimator}

Given the measurements in (1), the ML estimate of distance between nodes $i$ and $j$ is derived as:

$$
\hat{d}_{i j}=d_{0} 10^{\frac{L_{i j}-L_{0}}{10 \gamma}}
$$

We can reformulate (4) as:

$$
\mu_{i j} \hat{d}_{i j}^{2}=\eta d_{0}^{2}
$$

where $\mu_{i j}=10^{-\frac{L_{i j}}{5 \gamma}}$, and $\eta=10^{-\frac{L_{0}}{5 \gamma}}$. Assuming that the initial target positions estimates are available, from (5), target node $i$ updates its position estimate by minimizing the following LS problem:

$$
\boldsymbol{x}_{i}^{(k+1)}=\underset{\boldsymbol{x}_{i}}{\operatorname{argmin}} \sum_{(i, j) \in \mathcal{E}}\left(\mu_{i j}\left\|\boldsymbol{x}_{i}-\widehat{\boldsymbol{x}}_{j}\right\|^{2}-\eta d_{0}^{2}\right)^{2} .
$$

Define auxiliary variables $\varphi_{i}=\left\|x_{i}\right\|^{2}$ and $\boldsymbol{\alpha}=\left[\alpha_{i j}\right]$, where $\alpha_{i j}=\mu_{i j}\left(\varphi_{i}-\right.$ $\left.2 \widehat{\boldsymbol{x}}_{j}{ }^{T} \boldsymbol{x}_{i}+\widehat{\boldsymbol{x}}_{j}{ }^{T} \widehat{\boldsymbol{x}}_{j}\right)-\eta d_{0}^{2}, \forall(i, j) \in \mathcal{E}$. Introduce an epigraph variable $e$, and apply second-order cone constraint (SOCC) relaxation to obtain the following convex optimization problem:

$$
\underset{\boldsymbol{x}_{i}, \boldsymbol{\varphi}, \boldsymbol{\alpha}, \boldsymbol{e}}{\operatorname{minimize}} e
$$

subject to

$$
\begin{array}{r}
\alpha_{i j}=\mu_{i j}\left(\varphi_{i}-2 \widehat{\boldsymbol{x}}_{j}{ }^{T} \boldsymbol{x}_{i}+\widehat{\boldsymbol{x}}_{j}{ }^{T} \widehat{\boldsymbol{x}}_{j}\right)-\mu d_{0}^{2}, \forall(i, j) \in \mathcal{E}, \\
\|2 \boldsymbol{\alpha} ; e-1\| \leq e+1, \quad\left\|2 \boldsymbol{x}_{i} ; \varphi_{i}-1\right\| \leq \varphi_{i}+1 .
\end{array}
$$

Problem defined in (7) is a SOCP problem, which can be efficiently solved by the CVX package [15] for specifying and solving convex programs. We will refer to (7) as "SOCP" in the further text. 


\subsection{The Proposed Node Selection Mechanism}

Theoretically, it is possible that each target node communicates with every other node in the network, i.e., $\left|\Omega_{i}\right|=T+A-1$, for $i=1, \ldots, T$. In practice however, the size of the neighborhood fragments is much smaller, due to limited energy resources. Since the RSS range errors are multiplicative, i.e., they have constant multiplicative factors with range [1], edges from distant nodes may negatively influence the performance. Hence, we intend to drop all potentially bad links in the network.

Here, we investigate two possible node selection mechanisms that are based on distance criterion. The basic idea is to exploit the information of only $n_{d}$ closest neighbors at each target node, rather than the information from all $\left|\Omega_{\mathrm{i}}\right|$ neighbors. Thus, our goal is to achieve a good trade-off between the estimation accuracy and energy consumption. The derivation of the optimal mechanism is not possible [11]; hence, we use a sub-optimal scheme that is based on distance estimates between nodes. The scheme is suboptimal, since we deal with noisy RSS measurements, and the measurements with the highest RSS are not necessarily the best ones.

Target node $i$ estimates the distance between itself and its neighbor $j, j \in \Omega_{i}$, according to (4). We can sort these estimates of target node $i$ as:

$$
\hat{d}_{i_{1}} \leq \hat{d}_{i_{2}} \leq \cdots \leq \hat{d}_{i_{m}}, \quad 1, \ldots, m \in \Omega_{i},
$$

where $\hat{d}_{i_{1}}$ and $\hat{d}_{i_{m}}$ are the lowest and highest distance estimates, respectively. Target node $i$ selects the new group of cooperating nodes, $\Omega_{i}^{\prime}$ as follows:

1) Choosing all anchors and $n_{d}$ nearest target nodes, i.e., $\Omega_{i}^{\prime}=\{j: j \in \mathcal{A}\} \cup$ $\left\{i_{1}, i_{2}, \ldots, i_{n_{d}}: i_{m} \in \mathcal{T}\right.$, for $\left.m=1, \ldots, n_{d}\right\}$;

2) Choosing $n_{d}$ nearest neighbors, i.e., $\Omega_{i}^{\prime}=\left\{i_{1}, i_{2}, \ldots, i_{n_{d}}\right\}$.

Applying the node selection mechanism is likely to reduce the energy consumption inside a WSN, as it can be seen in Fig. 1.

Table 1. Summary of the Considered Algorithms

\begin{tabular}{|c|c|c|}
\hline Algorithm & Description & Complexity \\
\hline DSCL & $\begin{array}{c}\text { The spatially constrained algorithm in } \\
{[10]}\end{array}$ & $K_{\max } \times T \times F \times \mathcal{O}\left(\max _{i}\left\{\left|\Omega_{i}\right|\right\}\right)$ \\
\hline LS & The least squares algorithm in [11] & $K_{\max } \times T \times t_{i t e r 2} \times \mathcal{O}\left(\max _{i}\left\{\left|\Omega_{i}\right|\right\}\right)$ \\
\hline SOCP & The proposed algorithm in (7) & $K_{\max } \times T \times \mathcal{O}\left(\left(\max _{i}\left\{\left|\Omega_{i}\right|\right\}\right)^{3}\right)$ \\
\hline
\end{tabular}

Fig. 1 illustrates a possible network layout when target nodes cooperate with (a) all nodes in their communication range, (b) all anchor neighbors and at most $n_{d}$ nearest target neighbors, and (c) at most $n_{d}$ nearest neighbors. One can see from Fig. 1 that the network is significantly skimmed when the node selection mechanism is applied. Although this procedure may degrade the estimation accuracy, it is likely to preserve energy in a WSN in both communication and computation phase of the algorithm. 


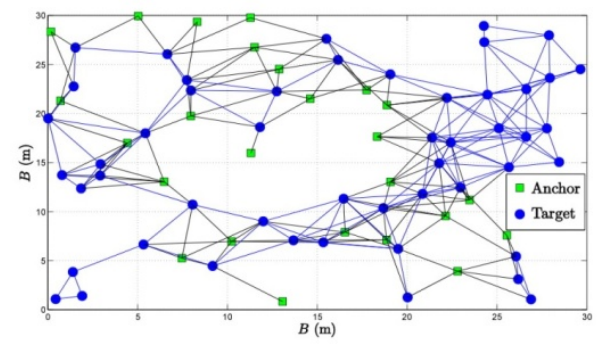

a)

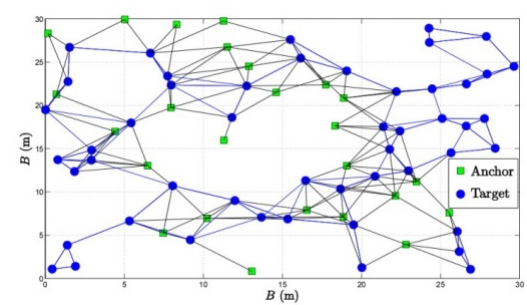

b)

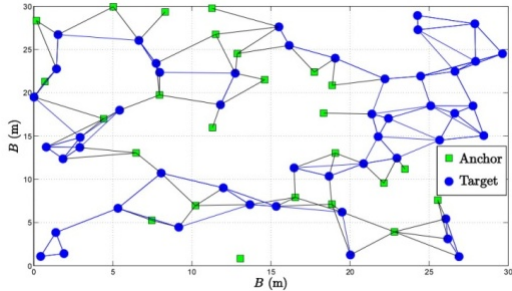

c)

Fig. 1. A possible network layout of a WSN with $A=25, T=50$ nodes. Target nodes communicate with a) all nodes in their communication range, b) all anchor neighbors and at most $n_{d}=3$ nearest target neighbors, and c) at most $n_{d}=4$ nearest neighbors.

\section{Analysis of Computational Complexity}

The trade-off between the computational complexity and estimation accuracy determines the applicability potential of an algorithm; hence, it is the most important feature of a localization algorithm. For this reason, we are also interested in comparing the computational complexities of the proposed approach with the existing ones. Here, we investigate the worst case asymptotical complexity of the algorithms, i.e., we present only the dominating elements.

Table 1 gives an overview of the considered algorithms, together with their computational complexities. The worst case complexity of the proposed SOCP approach is calculated according to [16].

From Table 1 it can be seen that the worst case complexity of a distributed algorithm mainly depends on the neighborhood fragments (the biggest one). The size of the neighborhood fragments is not severely affected if the number of nodes in the network is increased, which makes the distributed algorithms a desirable solution in highly-dense or large-scale networks. Furthermore, we can see from Table I that the new approach is computationally the most demanding. This fact is justified by its superior performance in terms of the estimation accuracy and convergence, as we will see in the following section. 


\section{Performance Assessment}

In this section, we present the computer simulation results in order to compare the performance of the proposed approach with the state-of-the-art. The new approach was solved by using the MATLAB package CVX [15], where the solver is SeDuMi [17].

To generate the RSS measurements, the propagation model (1) is used. We considered a random deployment of nodes inside a square region of length $B=30 \mathrm{~m}$ in each Monte Carlo $\left(M_{c}\right)$ run. Random deployment of nodes is of practical interest, since the algorithms are tested against various network topologies. In order to make the comparison fair, we first obtained $M_{c}=500$ nodes positions, and we run the considered algorithms for these scenarios. Furthermore, we made sure that the network graph was connected in each $M_{c}$ run. The path loss exponent is $\gamma=3$, the reference distance $d_{0}=1 \mathrm{~m}$, the reference path loss $L_{0}=40 \mathrm{~dB}$, and the communication range of a node $R=B / 5 \mathrm{~m}$. We assumed that the initial estimation of the target positions, $\widehat{\boldsymbol{X}}^{(0)}$, is in the intersection of the diagonals of the square area, and the maximum number of iterations $K_{\max }=100$. We assumed that the working sequence of the nodes is random, and that all target nodes use the last received position estimate of their neighbors. A iteration is completed when all $T$ target nodes compute and transmit their position estimates. As the performance metric we used the normalized root mean square error (NRMSE), defined as

$$
\mathrm{NRMSE}=\sqrt{\frac{1}{T M_{c}} \sum_{i=1}^{M_{C}} \sum_{j=1}^{T}\left\|x_{i j}-\widehat{x}_{i j}\right\|^{2}},
$$

where $\widehat{\boldsymbol{x}}_{i j}$ denotes the estimate of the true location of the $j$-th target in the $i$-th Monte Carlo run, $\boldsymbol{x}_{i j}$.

Fig. 2 illustrates the NRMSE versus $k$ performance of the considered algorithms, when target nodes choose all neighboring anchor nodes and at most $n_{d}$ nearest target nodes for cooperation. As the lower bound on the performance, we have provided the simulation results when the considered approaches use all nodes that are inside their communication range for cooperation. In Fig. 2, the average number of anchor neighbors was $\bar{n}_{i a}=2.54$, and the average number of all neighbors was $\bar{n}_{i}=7.48$. It is worth noting that the new approach outperforms the state-of-the-art for more than 1 $\mathrm{m}$, in the case where no node selection mechanism was applied. From Fig. 2, we can see that the performance of all approaches improves when the number of cooperating target neighbors increases, as expected. Although the proposed approach does not converge for $n_{d}=1$, it outperforms the existing ones in general. This can be seen from Fig. 2 (top), where the proposed approach reaches the lower bound for $n_{d} \geq 3$, while the existing ones show considerable gap for all choices of $n_{d}$ in comparison to their lower bounds, Figs. 2 (middle and bottom).

Fig. 3 illustrates the NRMSE versus $k$ performance of the considered algorithms, when target nodes choose at most $n_{d}$ nearest neighboring nodes for cooperation. In Fig. 3 , the average number of neighbors was $\overline{n_{l}}=7.48$. From Fig. 3 , it can be seen that the performance of all algorithms betters as the number of cooperating nodes is 
increased. This result is anticipated, because when $n_{d}$ is increased the collected information of each target node also increases, as well as the probability of having more anchor neighbors. Moreover, we can see that the new approach outperforms the existing ones for $n_{d}>3$, requiring only $k=30$ iterations to converge in general.
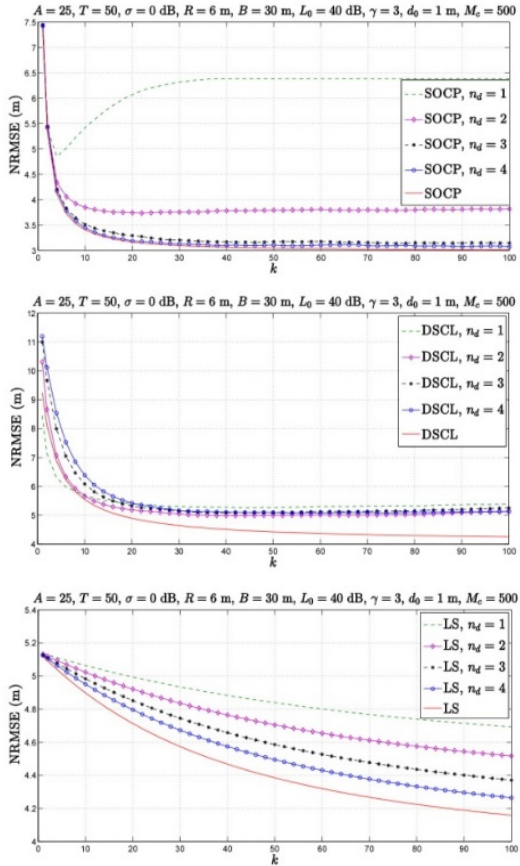

Fig. 2. NRMSE versus $k$ comparison of the proposed SOCP (top), DSCL (middle), and LS (bottom) approach for different $n_{d}$ nearest target neighbors, when $A=25, T=50, \sigma=$ $0 \mathrm{~dB}, R=6 \mathrm{~m}, B=30 \mathrm{~m}, L_{0}=40 \mathrm{~dB}, \gamma=$ $3, d_{0}=1 \mathrm{~m}, M_{c}=500$.
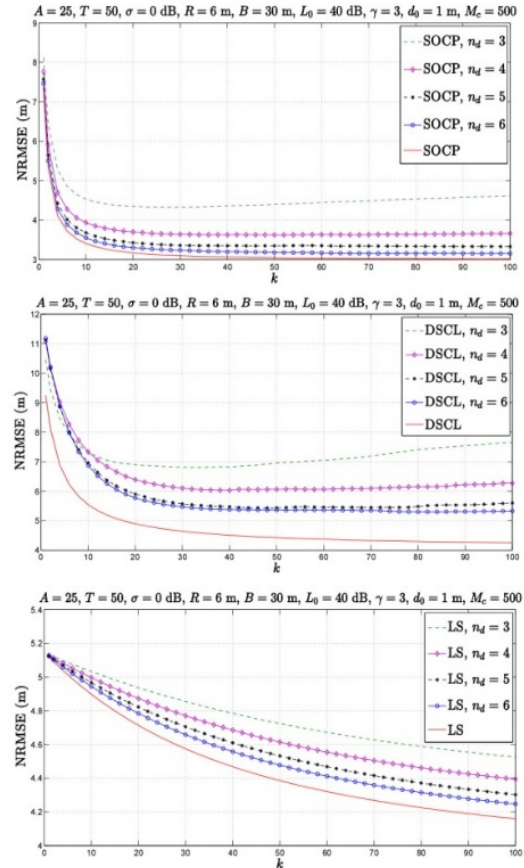

Fig. 3. NRMSE versus $k$ comparison of the proposed SOCP (top), DSCL (middle), and LS (bottom) approach for different $n_{d}$ of the nearest neighbors, when $A=25, T=$ $50, \sigma=0 \mathrm{~dB}, R=6 \mathrm{~m}, B=30 \mathrm{~m}, L_{0}=$ $40 \mathrm{~dB}, \gamma=3, d_{0}=1 \mathrm{~m}, M_{c}=500$.

\section{Conclusions}

In this work, we investigated the RSS-based target localization problem in large-scale cooperative WSN. We proposed a completely distributed algorithm based on SOCP relaxation technique. The simulation results confirm the superiority of the proposed approach in terms of the estimation accuracy and convergence. Furthermore, we have considered the case where the target nodes limit the number of cooperating nodes, by selecting only a certain number of neighbors with the highest RSS measurements. This simple procedure does not affect the computational complexity of an algorithm, and it can reduce the energy consumption inside the network. Unlike the existing 
approaches, the simulation results show that the proposed method does not deteriorate significantly in the performance when the number of cooperating nodes is reduced.

In this paper, we have presented the results accumulated via computer simulations. The algorithm's implementation in a real WSN is yet to be done, and practical indoor scenarios will be of particular interest.

Acknowledgments. This work was partially supported by Fundação para a Ciência e a Tecnologia under Projects PEst-OE/EEI/UI0066/2014, EXPL/EEI-TEL/0969/2013-MANY2 COMWIN and EXPL/EEI-TEL/1582/2013 GLANC, PEst-OE/EEI/LA0008/2013 (IT pluriannual founding and HETNET), PEst-OE/EEI/UI0066/2011 (UNINOVA pluriannual founding), EnAcoMIMOCo EXPL/EEITEL/2408/2013, ADIN PTDC/EEI-TEL/2990/2012, and TR 32043, and the grant SFRH/BD/91126/2012 and Ciência 2008Post-Doctoral Research grant.

\section{References}

1. Patwari, N.: Location Estimation in Sensor Networks. PhD Thesis, University of Michigan, Ann Arbor, MI, USA (2005)

2. Destino, G.: Positioning in Wireless Networks: Noncooperative and Cooperative Algorithms. PhD Thesis, University of Oulu, Oulu, Finland (2012)

3. Patwari, N., Ash, J.N., Kyperountas, S., Hero III, A.O., Moses, R.L., Correal, N.S.: Locating the Nodes: Cooperative Localization in Wireless Sensor Networks. IEEE Signal Process. Mag. 22(4), 54-69 (2005)

4. Ouyang, R.W., Wong, A.K.S., Lea, C.T.: Received Signal Strength-based Wireless Localization via Semidefinite Programming: Noncooperative and Cooperative Schemes. IEEE Trans. Veh. Technol. 59(3), 1307-1318 (2010)

5. Wang, G., Yang, K.: A New Approach to Sensor Node Localization Using RSS Measurements in Wireless Sensor Networks. IEEE Trans. Wirel. Commun. 10(5), 1389-1395 (2011)

6. Wang, G., Chen, H., Li, Y., Jin, M.: On Received-Signal-Strength Based Localization with Unknown Transmit Power and Path Loss Exponent. IEEE Wirel. Commun. Lett. 1(5), 536-539 (2012)

7. Vaghefi, R.M., Gholami, M.R., Buehrer, R.M., Ström, E.G.: Cooperative Received Signal Strength-Based Sensor Localization With Unknown Transmit Powers. IEEE Trans. Signal Process. 61(6), 1389-1403 (2013)

8. Salman, N., Ghogho, M., Kemp, A.: H: On the Joint Estimation of the RSS-based Location and Path-loss Exponent. IEEE Wireless Commun. Lett. 1(1), 34-37 (2012)

9. Béjar, B., Zazo, S.: A Practical Approach for Outdoors Distributed Target Localization in Wireless Sensor Networks. EURASIP J. Adv. Signal Process. 1-11 (May 2012)

10. Cota-Ruiz, J., Rosiles, J.G., Rivas-Perea, P., Sifuentes, E.: A Distributed Localization Algorithm for Wireless Sensor Networks Based on the Solution of Spatially-Constrained Local Problems. IEEE Sens. J. 13(6), 2181-2191 (2013)

11. Bel, A., Vicario, J.L., Seco-Granados, G.: Localization Algorithm with On-line Path Loss Estimation and Node Selection. Sensors 11(7), 6905-6925 (2011)

12. Rappaport, T.S.: Wireless Communications: Principles and Practice. Prentice-Hall, Upper Saddle River (1996) 
13. Sichitiu, M. L., Ramadurai, V.: Localization of wireless sensor networks with a mobile beacon. In: Proceedings of the IEEE International Conference on Mobile Ad-hoc and Sensor Systems (MASS), Fort Lauderdale, FL, USA, pp. 174-183 pp. 25-27, October 2004

14. Boyd, S., Vandenberghe, L.: Convex Optimization. Cambridge University Press, Cambridge (2004)

15. Grant, M., Boyd, S.: CVX: Matlab Software for Disciplined Convex Programming. Version $1.21 \mathrm{http} / / / \mathrm{cvxr} . c 0 \mathrm{cov} / \mathrm{cvx}$ (accessed on April 15, 2010)

16. Pólik, I., Terlaky, T.: Interior Point Methods for Nonlinear Optimization. In: Di Pillo, G., Schoen, F. (eds.) Nonlinear Optimization, 1st ed. Springer, Heidelberg, Cetraro, Italy (2010)

17. Sturm, J.F.: Using SeDuMi 1.02, a MATLAB Toolbox for Optimization Over Symmetric Cones. Optim. Meth. Softw. 11, 625-653 (1999) 